\title{
NATURAL AND EXPERIMENTAL EVIDENCE OF VISCEROTROPIC INFECTION CAUSED BY LEISHMANIA TROPICA FROM NORTH SINAI, EGYPT
}

\author{
By \\ SAID A. DOHA ${ }^{1^{*}}$, MAGDI G. SHEHATA ${ }^{2}$, ADEL R. FAHMY $^{2}$ \\ AND ABDALLAH M. SAMY2,3
}

Research and Training Center on vectors of diseases, Faculty of Science building, Ain Shams University ${ }^{1}$, Entomology Department, Faculty of Science, Ain Shams University, Cairo 11566, Egypt ${ }^{2}$, and Biodiversity Institute and Department of Ecology and Evolutionary Biology, University of Kansas, Lawrence, Kansas 66045 USA $^{3}$

( ${ }^{*}$ Correspondence Dr. Doha, doha57@yahoo.com)

\begin{abstract}
Cutaneous leishmaniasis (CL) is a neglected clinical form that is quite prevalent in Eastern North parts of the country in Sinai Peninsula. Leishmania tropica was identified by previous reports as the causative agent responsible for viscerotropic infections in patients and experimental animals. Here, we reported the viscerotropic infections from naturally infected rodent Gerbillus pyramidum floweri collected from North-Sinai. Footpad and tail lesions, spleenomegaly, and malformed dark-colored spleen were the characteristic CL symptoms. The spleen of the rodent found positive to amastigote impression smear. ITS-1 DNA was sequenced and revealed $100 \%$ identity of the strain in the current study to the other L. tropica sequences identified from the patients with the suspected CL and inhabited the same study area. The current findings confirmed the susceptibility of gerbil to L. tropica, and raise the concerns for the role of rodents as accidental host suffering the infections. The susceptibility of wild and experimental rodents to the same L. tropica strain was also investigated; BALB/c and G. pyramidum were more susceptible to $L$. tropica $(24.33 \pm 4.37$ and $25 \pm 4.58$ days post-infection, respectively). Similar viscerotropic pathologies were reported in experimental infection of only golden hamster $(\approx 120$ days post-infection), and G. p. floweri $(\approx 160$ days post-infection).
\end{abstract}

Key words: Viscerotropic, L. tropica, North Sinai, ITS-1 DNA, Splenomegaly

\section{Introduction}

The characteristic CL lesions vary between and within regions, reflecting different species of parasite, or the type of zoonotic cycle concerned, and immunological status of the patients (WHO, 2010). In Egypt, CL is caused by both the Leishmania major and L. tropica (Shehata et al, 2009). L. major usually presents as open ulcers starts as a papule or nodule at the site of inoculation; however, multiple ulcers can also be reported. L. major is known to be transmitted by Phlebotomus papatasi, while the vector of CL caused by L. tropica remains unknown in the Sinai Peninsula.

L. tropica was recently identified as the pathogen responsible for two CL cases in Sinai, and the parasite strains were also identified from the mammalian host Gerbillus pyramidum (Shehata et al, 2009). The later report for L. tropica from Egypt proposed two scenarios for the incursion of $L$. tropica; 1) historical difficulties in distinguishing L. major from L. tropica (Jacobson, 2003) have masked the presence of $L$. tropica in past studies, or 2) recent incursion by $L$. tropica from adjacent endemic regions may be responsible for the presence of $L$. tropica. In Egypt, the knowledge about this species is still underestimated with few known and well characterized details about the transmission of L. tropica across Sinai (Shehata et al, 2009).

Cutaneous leishmaniasis caused by $L$. tropica was previously known as urban anthropontotic CL, with no evidence for the presence of the zoonotic transmission cycle, 
however, the parasite was identified in several studies from the zoonotic foci in Kenya, Israel, Palestine, and Egypt (Sang et al, 1994; Jacobson et al, 2003; Shehata et al, 2009). Viscerotropic infection caused by $L$. tropica was reported in patients returned from Saudi Arabia and Afghanistan (i.e. leishmaniasis recidivans; Magill et al, 1993), and from experimentally infected animals (Lira et al, 1998; Mahmoudzadeh-Niknam et al, 2007), however, little is known about visceral infections in wild rodents.

Here, the present study reports the evidence of viscerotropic infection from naturally infected $G$. p. floweri collected from El Barth community, Northern Sinai, Egypt and identified insights for the animal reservoirs associated with the disease transmission. The current results do not reflect the primary objectives of the study to identify the sand fly vectors, and animal reservoirs, but, referred only to the surprising findings of the viscerotropic infections and the experimental infections trials of $L$. tropica from Egypt.

\section{Materials and Methods}

The study was carried out in El Barth village $\left(31^{\circ} 01^{\prime} \mathrm{N}, 34^{\circ} 12^{\prime} \mathrm{E}\right.$ to $30^{\circ} 8^{\prime} \mathrm{N}, 34^{\circ}$ $\left.17^{\prime} \mathrm{E}\right)$ located in northeastern Sinai Peninsula, about $35 \mathrm{~km}$ southeast of Rafah on the Egyptian borders with Palestine and is unique in a way that it can be considered one of the most remote areas from Cairo and Nile Delta. El Barth is located on El-Goora road and is divided into four sectors namely Kilo25 ( $\left.31^{\circ} 01^{\prime} \mathrm{N}, 34^{\circ} 12^{\prime} \mathrm{E}\right), \mathrm{Kilo} 30\left(31^{\circ}\right.$ $\left.00^{\prime} \mathrm{N}, 34^{\circ} 14^{\prime} \mathrm{E}\right)$, Kilo33 (30 $59^{\circ} \mathrm{N}, 34^{\circ} 15^{\prime}$ E), and Kilo36 ( $\left.30^{\circ} 8^{\prime} \mathrm{N}, 34^{\circ} 17^{\prime} \mathrm{E}\right)$. The area is characterized by the presence of sand dunes of varying elevations enclosing sparsely vegetated areas (Fig. 1 A, B). El Barth is inhabited principally by Bedouins and has approximately 5,500 inhabitants; however, temporary workers from different governorates were reported during the time of the study.

Sand fly collection was carried out using the sticky paper traps (Rioux et al, 1982) for eight nights in December 2006. Twenty five sticky traps were used for each collection site (2 collection sites/sector). The study used sticky paper traps to target the areas around the rodent burrows distributed in the area. Traps were set before sunset and recovered the next morning. Recovered sticky traps were placed in labeled plastic bags, and transported to a temporary field laboratory. Flies were cleaned in chloral hydrate: phenol $(1: 1 \mathrm{v} / \mathrm{v})$ and then mounted in Puri's medium (Smart et al, 1965) for species identification via the local morphological keys (Lane, 1986).

Rodents were trapped using wire-box rodent traps (Morsy et al, 1992; Hamadto et al, 2007; Fig. 1 C). The traps were placed adjacent to the rodent burrows for eight nights during December 2006. The field collected rodents were identified using the local taxonomic keys (Osborn and Helmy, 1980), and then maintained for at least 6 months to monitor the lesion development. All rodents died during the collection were dissected for Leishmania culturing trials and parasite identification. Full-thickness punch-biopsies were removed from the suspected lesions of the rodents with the characteristic Leishmania lesions. The rodents tested positive to amastigote impression smear were anaesthetized, and biopsy samples were then obtained from spleen. The biopsy sample was divided into two portions; one for Giemsa impression smear to detect the presence or absence of Leishmania amastigotes, and the second portion was used for parasite culturing in NNN-medium and frequent diagnostic tests.

The identity of the strain reported by this study was first identified by real time PCR, and RFLP (Shehata et al, 2009). Here, the parasite was centrifuged for PCR, DNA sequencing as previously described by El Tai et al. (2000) and applied by Shehata et al. (2009). Simply, approximately $25 \mu \mathrm{L}$ of the culture pellet was transferred to a sterile 1.5 $\mathrm{mL}$ tube, extracted as per protocol instructions, and eluted in $100 \mu 1$ elution buffer. 


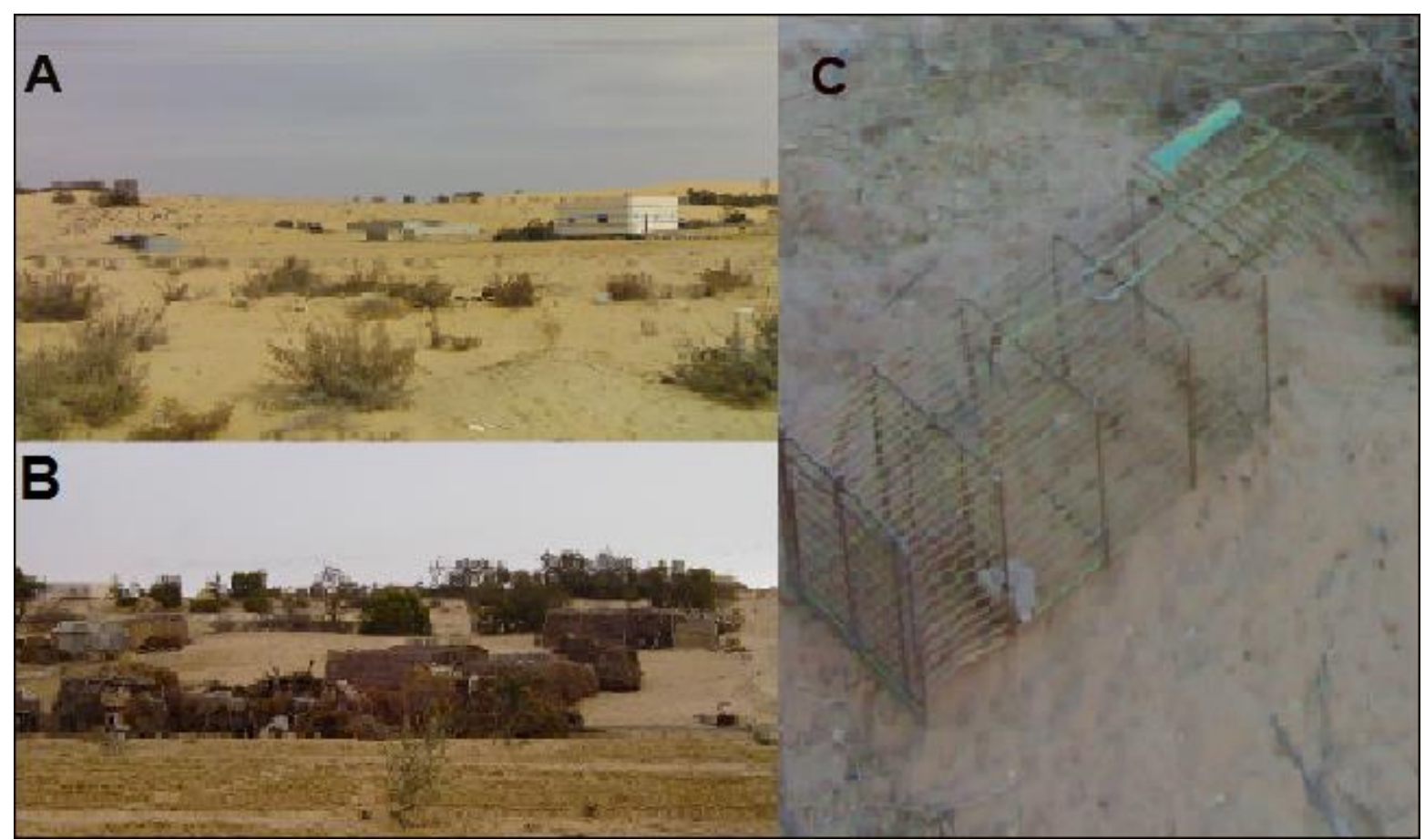

Fig. 1: Study area where sand fly and rodents collected; a) sandy habitat and sparse vegetation, b) cereals stores with wire-box rodent trap

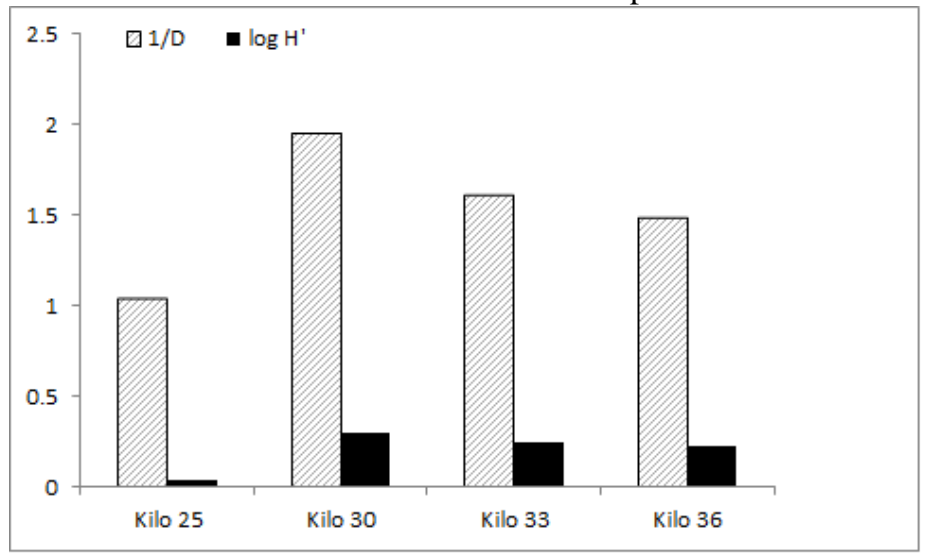

Fig. 2: Biodiversity index of both sand flies collected from El Barth community, North Sinai, Egypt. Biodiversity Simpson (1/D) and Shannon (H') indices estimated from sand fly data collected in December 2006.

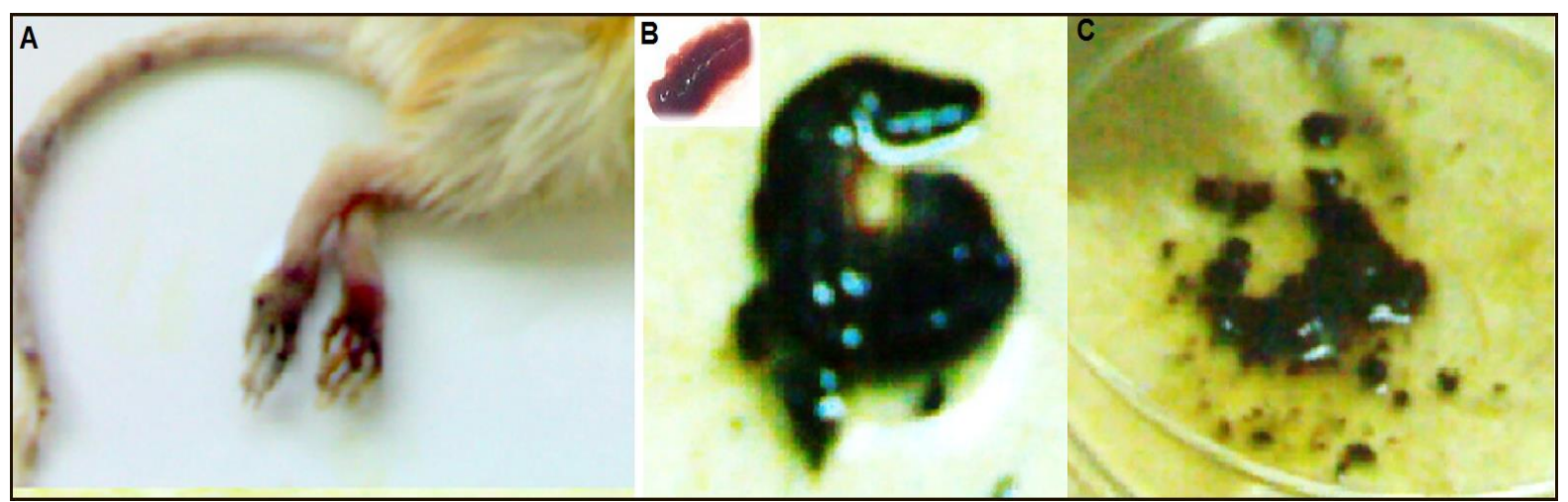

Fig. 3: Progressive and characteristic Leishmania lesions on rodent G. pyramidum floweri collected from El Barth community at different parts of body; A) footpads and tail lesions, b) enlarged spleen with normal spleen on the top, and c) spleen before culturing. 
Proteinase $\mathrm{K}$ digestion was performed overnight at $56^{\circ} \mathrm{C}$. The ribosomal internal transcribed spacer 1 (ITS1) was amplified from the spleen isolate by conventional PCR, and sequenced in both directions from the same isolate using the primer pair L5.8S and LITSR (El Tai et al, 2000). MEGA 6 was used to query GenBank, and for DNA sequence analysis (Tamura et al, 2013). The DNA sequence from spleen isolate and other homologs from GenBank were aligned for gene tree construction in MEGA 6.

L. tropica isolate originates from a naturally infected rodent was divided into two portions; one was inoculated to NNN medium, and the second portion was inoculated to the footpad of healthy Syrian hamster Mesocricetus auratus. The promastigotes obtained from NNN medium were inoculated twice at 3 days intervals to $5 \mathrm{ml}$ of Schneider's Drosophila cell culture medium supplemented with the $10 \%$ fetal calf serum (Sigma, Saint Louis, MO, USA, and Gibco-BRL, Gaithersburg, MD, USA) for parasite mass rearing before experimental infections. On the $7^{\text {th }}$ day, the medium was centrifuged at $1,500 \mathrm{~g}$ for 5 minutes. Promastigotes were obtained from sediment, and washed with sterile phosphate buffer suspension containing L. tropica at a density of $2 \times 10^{7}$ promastigotes/ml was inoculated subcutaneous to the posterior footpad of six experimental animals of no history for CL (three BALB/c, three golden hamster), and six wild rodents (rhree Gerbillus andersoni, and three G. p. floweri). These animals were checked weekly post-infection (PI) for any characteristic CL lesions (at the inoculation site) PI.

Finally, the size of the footpad lesions of the right hind feet was measured as increase in footpad thickness after subtracting the size of the uninfected footpad on the left hind feet. The thickness was determined by a digital caliper (Mitutoyu, Kawasaki, Kanagawa, Japan).

The footpad thickness increased due to the infection was defined as the difference between the thickness of the infected footpad and the thickness of the uninfected contralateral footpad.

\section{Results}

An overall total of 819 sand flies were collected from different sectors during the study (Tab. 1) in 8 nights using 1600 sticky paper traps. Males comprised 59.2\% ( $\mathrm{N}=$ 485 ) of the catch (Female/male ratio of 1:1.45). All of flies were collected from around and close to the rodent burrows.

These flies represented two species of one genus; Phlebotomus (Phlebotomus) papatasi (Scopoli), and P. (Paraphlebotomus) sergenti (Parrot). P. papatasi was the most predominant species and represented $76.7 \%$ $(\mathrm{N}=628)$. The sand fly population diversity were more significant in kilo 30 (Simposon index $=0.51$, Shannon index $\mathrm{H}=0.68$; Fig. 2). The Sex ratios revealed the presence of more males in all collections except in case of $P$. sergenti from Kilo 36 of the study area, where, more females $(\mathrm{N}=23)$ were collected than males $(\mathrm{N}=17)$.

Eighteen rodents were collected from the study sites. Three rodent species were identified; Gerbillus pyramidum floweri $(\mathrm{N}=11)$ G. andersoni $(\mathrm{N}=7)$, and Mus musculus $(\mathrm{N}=1)$. Rodents were maintained at the (RTC) and Training Center on Vector of Diseases for at least 6 months for the characteristic Leishmania lesions. Rodents with the characteristic Leishmania lesions (Fig. 3 A, B) were examined for the presence of amastigotes using Giemsa impression smear. Leishmania amastigotes were detected in two G. p. floweri, and one G. andersoni. The spleen of only one G. p. floweri was found infected with Leishmania amastigote.

The spleen of infected animal showed splenomegaly, malformation, and was darkcolored (Fig. 3 A, and C). These abnormalities were the main characteristics that differentiate the infected and the normal spleen. The parasite loads of the infected spleen were much higher than $2 \times 10^{7}$ parasites $/ \mathrm{ml}$, and the spleen was enlarged due to the increase of these loads (Fig. 3 B, C). 
The ITS-1 sequence were obtained for Leishmania isolate collected from rodent spleen (accession KC822364) and BLAST results indicate that they are most similar to L. tropica strains originally identified from a patient (MHOM/EG/06/RTC-66; FJ460457) and wild animal (MGER/EG/06/ RTC-73: accession FJ460458) collected in previous studies (Shehata et al, 2009). The isolate shared $97 \%$ identity to other isolates from the Sudan (MHOM/SU/60/OD: accession EU326226), and Afghanistan (MHOM/ AF /88/KK27; accession GQ913688). A gene tree constructed from the identified sequence, and other 23 homologues from the GenBank, showed definitive clustering of the spleen isolate with the other L. tropica sequences identified from Sinai (Fig. 4).

There was no significant difference between the responses of different species to $L$. tropica ( $P>0.5$; Fig. 5 A). BALB/c mice was more susceptible to L. tropica strain relative to the golden hamster based on the time required for the first appearance of the characteristic Leishmania lesions; characteristic CL lesions were developed 24.33 \pm 4.37 $\& 28.67 \pm 4.41$ days PI in BALB/c mice, and golden hamsters, respectively.
On the other hand, similar lesions were developed $25 \pm 4.58$, and $37.67 \pm 3.53$ days PI in G. pyramidum, and G. andersoni, respectively. $G$. andersoni was the most resistant to infection based on the time required to develop the characteristics CL lesions. Positive amastigote smears were detected in all animals with the characteristic CL lesions $(\mathrm{N}=12)$, however, the spleen and liver infections were reported in only three G. p. floweri, one G. andersoni, $1 \mathrm{BALB} / \mathrm{c}$, and three golden hamster. Splenomegaly was reported only in hamster $(\mathrm{N}=3)$, and $G$. p. floweri $(\mathrm{N}=2)$ within 120 , and 160 days PI. The development of the spleen infections was similar to that developed and reported in the naturally infected G. p. floweri collected from the study area (Fig. 4).

In L. tropica-infected BALB/c mice, the footpad thickness increased only up to 120 days PI (Fig. 5 B), and then decreased in both mice infected with amastigotes or promastigotes. Later, the thickness decreases continue in the rodents infected with the amastigotes with a slight increase in the footpad diameter in 190 days PI. On the other hand, the mice infected with the promastigotes showed a decrease in the lesion thickness, with a slight increase in 160 days.

Table 1: Sand fly collected from and around rodent burrows at different sectors of El-Barth community.

\begin{tabular}{lcccccccc}
\hline & \multicolumn{4}{c}{$P$. papatasi } & \multicolumn{5}{c}{ P. sergenti } \\
\cline { 2 - 9 } Study sector & Male & Female & Total & F/M & Male & Female & Total & F/M \\
\cline { 2 - 9 } Kilo 25 & 107 & 53 & 160 & 0.50 & 3 & 0 & 3 & 0 \\
Kilo 30 & 73 & 46 & 119 & 0.63 & 51 & 32 & 83 & 0.63 \\
Kilo 33 & 112 & 82 & 194 & 0.73 & 39 & 26 & 65 & 0.67 \\
Kilo 36 & 83 & 72 & 155 & 0.87 & 17 & 23 & 40 & 1.35 \\
Grand total & 375 & 253 & 628 & 0.67 & 110 & 81 & 191 & 0.74 \\
\hline
\end{tabular}




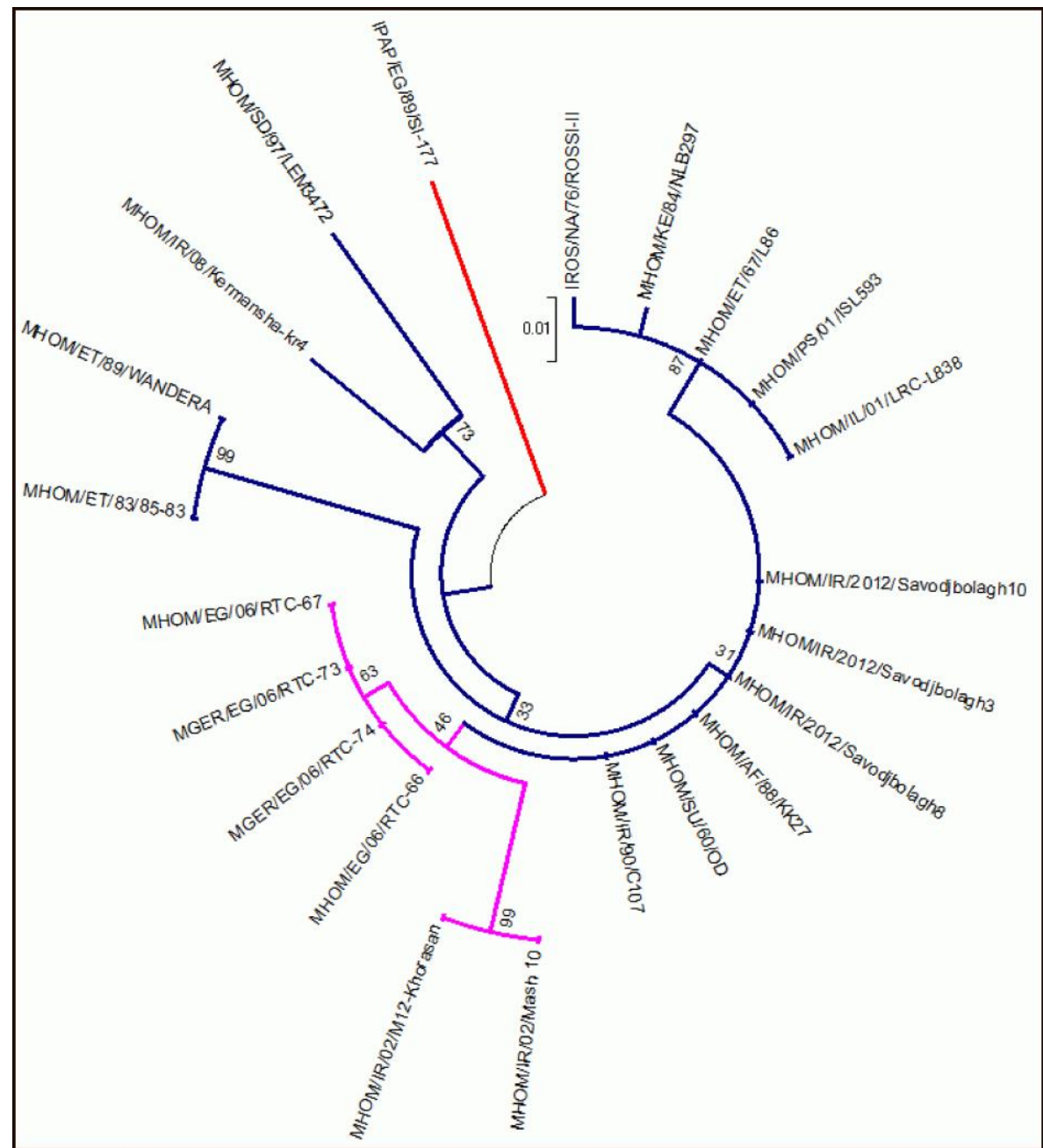

Fig. 4: Dendrogram inferred using maximum likelihood method of twenty three Leishmania strains collected from different areas including four L. tropica from Sinai, Egypt. Tree drawn to scale, with branch lengths measured in number of substitutions/ site. Analysis involved 23 nucleotide sequences. All positions containing gaps and missing data were eliminated. Total of 212 positions in final dataset. Evolutionary analyses were conducted in MEGA-6. L major from Sinai was used as outgroup.

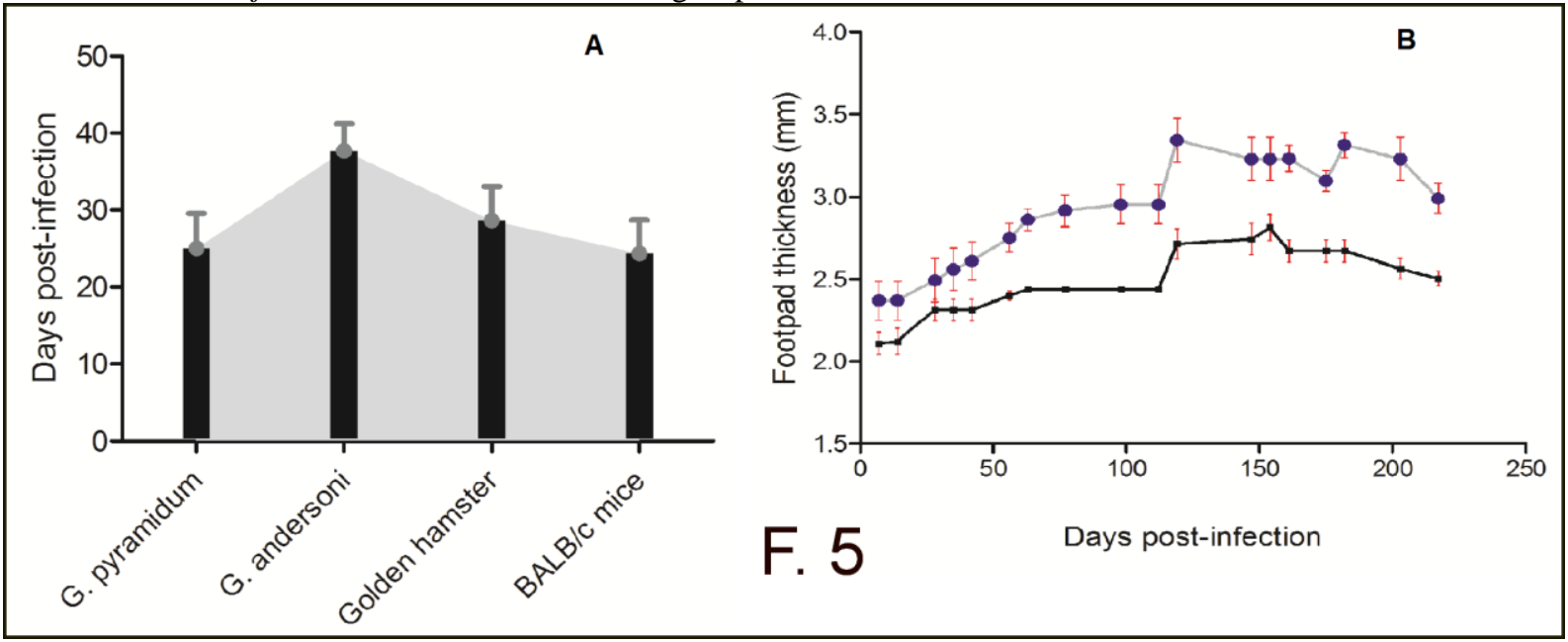

Fig. 5: Response of wild and experimental animals to L. tropica; a) Development of L. tropica in different animals. b) Course of lesion development in BALB/c mice after subcutaneous inoculation of $2 \times 10^{7}$ of $L$. tropica (MGER/EG/06/RTC-74) amastigotes (gray line), and promastigotes (Black line). Error bars in curves show standard error of mean of three mice used for experiment per each animal species. 


\section{Discussion}

The primary goal of this study was to investigate the role of the rodent populations in the CL transmission in one of the most recent focus identified in Sinai (Shehata $e t$ $a l, 2009)$. A part of our understanding to the disease ecology is to identify the sand fly species associated with the disease transmission in the study area. In this study, two sand fly species were identified; $P$. papatasi, and $P$. sergenti. $P$. papatasi, the potential vector of $L$. major in the Sinai Peninsula (Wahba et al, 1990; Shehata et al, 2009; Kassem et al, 2012) was the most prevalent sand fly in the present catches. The collections included both human populations (in a concurrent study), and mammalian reservoirs to identify the reservoir animals associated with the disease transmission in Sinai.

The verification of Leishmania parasite among the collected rodents is another important part in the study of the CL ecology. However, several studies reported different animal reservoirs (e.g. G. pyramidum and $P$. obesus) for cutaneous leishmaniasis caused by L. major in Sinai (Morsy et al, 1996; Shehata et al, 2009), but the role of these sylvan reservoirs in the transmission dynamics is still not well investigated. Similar observations were reported by other studies in Libya (Amro et al, 2012), and other neighboring countries, Israel and Palestine (Wasserberg et al, 2002) which have continuous disease outbreaks similar to that in Sinai, Egypt. For L. tropica transmission cycle, the disease dynamics is known to be anthroponotic (Morsy et al, 1991), however, the zoonotic transmission was documented in Kenya (Sang et al, 1994), Saudi Arabia (Morsy et al, 1997) and Israel (Svobodova et al, 2006; Talmi-Frank et al, 2010) where the rock hyrax was identified as the animal reservoirs. Recently, zoonotic transmission caused by L. tropica was reported in the same area of the current study (Shehata et al, 2009, Samy et al, 2010). Further investigations for the role of several animals collected from the study area demanded more efforts to identify the role of the sylvan reservoir for parasite circulation.

The three rodent species identified in this study were collected from and around the houses of the patients tested positive to CL (Ministry of Health, unpublished data). The infections were detected in only two $G$. pyramidum, and one G. andersoni. However, the current observations did not confirm what role is played by the two rodent species; if both are animal reservoirs or only accidental hosts? To incriminate a rodent as a reservoir host harboring the parasite, and maintains its transmission, it is necessary to demonstrate that the parasite populations require a particular mammal for the maintenance, and circulation in El-Barth community. However, there are needs for the rapid control interventions after the emergence of disease among hundreds of patients in such the small communities on the EgyptianPalestine border, but the study of the disease cycle demands also more extensive repeated studies on the sampled sites. This study was conducted to investigate the primary role played by the rodent populations to maintain the disease dynamics based on the fulfillment, and filling the gaps of five criteria reported by WHO (2010). The parasite was isolated from two rodent species collected from our study sites; G. pyramidum and $G$. andersoni, the parasite was found identical to that isolated from patients with the characteristic cutaneous leishmaniasis (Shehata et al, 2009; FJ460457), and the availability of the parasite in the skin to be taken up by the sand fly vector was also confirmed based on high density of the amastigotes in the amastigote impression smears of the footpad of the infected rodents. The later criteria were investigated in G. pyramidum and $G$. andersoni which harbored high parasite loads up to 120 days post-infection; however, there was difference in time between different treatments (promastigotes versus amastigotes) which might refer to the time of stage transformation of promastigotes to the amastigotes in the BALB/c mice. The 
rest of the criteria adopted by WHO was undertaken and recently developed in studies by RTC (Darwish et al, 2011) to illuminated unknown details for the disease transmission dynamics using the same parasite strains collected from our study.

This study provides an evidence for viscerotropic infections caused by $L$. tropica in the rodent $G$. $p$. floweri. Viscerotropic leishmaniasis is a comparatively mild form of visceral leishmaniaisis caused by $L$. tropica (Magill et al, 1993; Hyams et al, 2001; Soliman et al, 2006). The present data showed the same L. tropica strains with high loads in both skin and spleen infections of the rodent $G$. pyramidum. The growth patterns of L. tropica reported in this study is similar to the parasite growth patterns reported previously in human viscerotropic leishmaniasis, and caused by the Leishmania species identified from the Middle East (Magill et al, 1993). Several studies reported that visceralization and dissemination of Leishmania parasite into the spleen, as a visceral organ, is associated with a susceptible phenotype, whereas containment of the parasite in the skin and lymph nodes is associated with a resistant phenotype in animal (Laskay et al, 1995; Nicolas et al, 2000).

Mangoud et al. (2005) studied a total of 35 Egyptian parasitologically proven cutaneous leishmaniasis patients, the histopathological and immuno-histochemical picture. They concluded that the P53; S-phase fraction and DNA content must be in mind when dealing with a human cutaneous leishmaniasis and that early detection any nuclear mutation and cellular proliferation in the CL lesion(s) in order to avoid the development of the miserable and the complications of the skin cancer. Morsy (2013) examined skin biopsies from 65 parasitological proven cutaneous leishmaniasis patients from Egypt, Saudi Arabia, Jordan and Libya were histopathologically studied. The results showed that cutaneous leishmaniasis especially in hot areas pave the way to the mutation and development of skin can- cer. He added that anthroponotic cutaneous leishmaniasis (ACL) is known to cause single, self-healing and uncomplicated lesion mainly on the face. Basal cell carcinoma is a malignant epithelial neoplasm of skin that usually arises in areas of chronic sun exposure.

Based on such studies, the dissemination of the parasite to the spleen of G. pyramidum suggested that this animal is associated with susceptible phenotypes which suffers the infections, and develop similar pathologies to that reported in human (Magill et al, 1993). The visceralization in animals was not necessary to be fatal (Lira et al, 1998) but these progressive symptoms may cause the spleen necrosis that ends with death of animal. In our study, the rodent with the spleen infection could not survive for more than 24 hours after collection. This observation may confirm the role of $G$. pyramidum as an accidental host and not necessary to be a reservoir host which can harbor the parasite and play active role in transmission dynamics for a long time, and this may explain the low emergence of CL cases caused by $L$. tropica in Sinai with the break in the transmission cycle and the low vector density (Shehata et al, 2009; Fahmy et al, 2009). On the other hand, these observations raise a concern for the virulence of L. tropica strain collected from Sinai, and came in parallel agreement to the same conclusion by previous studies (Lira et al, 1998; Mahmoudzadeh-Niknam et al, 2007).

\section{Conclusion}

Further investigations of the development of the parasite in the human patients are recommended as one of the most important concern to be outlined in the study of CL in Sinai.

The possible visceralization in the rodents may reflect the actual role of the association of $G$. pyramidum as accidental host to Leishmania tropica, and raises the concerns for the presence of similar pathologies in Sinai Peninsula if the disease circulated for a long time in Egypt. 


\section{Acknowledgement}

The authors would like to acknowledge the Department of Entomology, and the Research and Training Center on Vectors of Diseases, Faculty of Science, Ain Shams Univerity for their support to this work. Thanks for comments by Professor Mohamed Kenawy, Entomology Department, Dr. Shabaan El Hossary, the Research and Training Center, and Dr. S. Soliman, Department of Zoology.

Financial support: Research and Training Center on Vectors of Diseases and the Department of Entomology, Ain Shams University. The project entitled "sand fly vectors and rodents reservoirs responsible for cutaneous leishmaniasis in a remote border area of Sinai, Egypt".

Disclaimer: The views expressed in this article are those of the authors and do not necessarily reflect the official policy or position of the Egyptian government, nor any of the authors' institutes. The experiments reported herein were conducted in compliance with the Animal Welfare Act and in accordance with the principles set forth in the Guide for the Care and Use of Laboratory Animals, Institute of Laboratory Animals Resources, National Research Council, National Academy Press and Council for International Organizations of Medical Sciences.

\section{References}

Amro, A, Gashout, A, Al-Dwibe, H, Zahangir, AM, Annajar, B, et al, 2012: First molecular epidemiological study of cutaneous leishmaniasis in Libya. PLoS Negl. Trop. Dis. 6, 6:e1700.

Darwish, AB, Tewfick, MK, Doha, SA, AboGhalia, AH, Soliman, BA, 2011: Vectorial competence of Phlebotomus papatasi (Diptera: Psychodidae) to transmit two old world Leishmania species: Leishmania major and L. tropica. J. Egypt. Soc. Parasitol. 41, 3:565-72.

El Tai, NO, Osman, OF, El Fari, M, Presber, W, Schönian, G, 2011: Genetic heterogeneity of ribosomal internal transcribed spacer (ITS) in clinical samples of Leishmania donovani spotted on filter paper as revealed by single-strand con- formation polymorphisms (sscp) and sequencing. Trans. R. Soc. Trop. Med. Hyg. 94:1-5.

Fahmy, AR, Samy, AM, Doha, SA, Shehata, MG, 2009. Preliminary field investigations on phlebotomine sandflies (Diptera: Psychodidae) from a recent cutaneous leishmaniasis focus in Northern-Sinai, Egypt. Egypt. Acad. J. Biol. Sci. 2, 1:9-15.

Hamadto, HA, Farrag, AB, Abdel-Maksoud, MK, Morsy, TA, 2007: Zoonotic cutaneous leishmaniasis: reservoir host and insect vector in north Sinai, Egypt. J. Egypt. Soc. Parasitol. 37, 3:843-50.

Hyams, KC, Riddle, J, Trump, DH, Graham, JT, 2001: Endemic infectious diseases and biological warfare during the Gulf War: a decade of analysis and final concerns. Am. J. Trop. Med. Hyg. 65, 5:664-70.

Jacobson, RL, Eisenberger, CL, Svobodova, M, Baneth, G, Sztern, J, et al, 2003: Outbreak of cutaneous leishmaniasis in northern Israel. J. Infect. Dis. 188:1065-73.

Jacobson, RL, 2003: Leishmania tropica (Kinetoplastida: Trypanosomatidae)-a perplexing parasite. Folia Parasitol. (Praha) 50:241-50.

Lane, RP, 1986: The sand flies of Egypt (Diptera: Phlebotominae). Bull. Br. Mus. Nat. Hist.

Entomol. 52:1-35.

Laskay, T, Diefenbach, A, Röllinghoff, M, Solbach, W, 1995: Early parasite containment is decisive for resistance to Leishmania major infection. Eur. J. Immunol. 25, 8:2220-7.

Lira, R, Méndez, S, Carrera, L, Jaffe, C, Neva, F, Sacks, D, 1998: Leishmania tropica: the identification and purification of metacyclic promastigotes and use in establishing mouse and hamster models of cutaneous and visceral disease. Exp. Parasitol. 89, 3:331-42.

Magill, AJ, Grogl, M, Gasser, RAJr, Sun, W, Oster, CN, 1993: Visceral infection caused by Leishmania tropica in veterans of operation Desert Storm. N. Engl. J. Med. 328:1383-7.

Mahmoudzadeh-Niknam, H, Kiaei, SS, Iravani, D, 2007: Viscerotropic growth pattern of Leishmania tropica in BALB/c mice is suggestive of a murine model for human viscerotropic leishmaniasis. Korean J. Parasitol. 45, 4:247-53.

Mangoud, AM, Sanad, EM, Fouad MAH, Morsy, TA, 2005: Proliferative changes of epidermal cells in lesions of cutaneous leishmaniasis. J. Egypt. Soc. Parasitol. 35, 3:761-72. 
Morsy, TA, 1996: Cutaneous leishmaniasis in Egypt: Review and comment. J. Egypt. Soc. Parasitol. 26, 1:105-30.

Morsy, TA, 2013: Cutaneous leishmaniasis predisposing to human skin cancer: forty years local and regional studies. J. Egypt. Soc. Parasitol. 43, 3:629 - 48

Morsy, TA, al Gahtani, YM, Faris, RM, 1991: Two abnormal cases of anthroponotic cutaneous leishmaniasis in Al Baha, Saudi Arabia. J. Egypt. Soc. Parasitol. 21, 3:675-8.

Morsy, TA, Merdan, AI, Wahba, MM, 1992: Leishmania infection sought in rodents in north Sinai, Egypt. J. Egypt. Soc. Parasitol. 22, 2:35763.

Morsy, TA, Sabry, AH, Rifaat, MM, Wahba, MM, 1996: Psammomys obesus Cretzschmar, 1828 and zoonotic cutaneous leishmaniasis in Sinai Peninsula, Egypt. J. Egypt. Soc. Parasitol. 26, 2:375-81.

Morsy, TA, al Dakhil, MA, el Bahrawy, AF, 1997: Natural Leishmania infection in rock hyrax, Procavia capensis (Pallas, 1766) order: Hyracoidea, trapped in Najran, Saudi Arabia. J. Egypt. Soc. Parasitol. 27, 1:75-81.

Nicolas, L, Sidjanski, S, Colle, JH, Milon, G, 2000: Leishmania major reaches distant cutaneous sites where it persists transiently while persisting durably in the primary dermal site and its draining lymph node: a study with laboratory draining lymph node: a study with laboratory mice. Infect. Immun. 68, 12:6561-6.

Osborn, DJ, Helmy, I, 1980: Mammals of Egypt. Chicago: Field Museum of Natural History, NAMRU-3, Cairo.

Rioux, JA, Périères, J, Killick-Kendrick, R, Lanotte, G, Bailly, M, 1982: Ecologie des leishmanioses dans le Sud de la France. 17. Echantillonnage des phlébotomes par le procédé des pièges adhésifs. Comparison avec la technique de capture sur appât humain. Ann. Parasitol. Hum. Comp. 57:631-5.

Samy, AM, Doha, SA, Fahmy, AR, Shehata, MG, 2010. First report of viscerotropic infection from naturally infected gerbil collected from North-Sinai, Egypt: Biennial meeting of the Royal Society of Tropical Medicine and Hygiene, Liverpool, United Kingdom.
Sang, DK, Njeru, WK, Ashford, RW, 1992: A possible animal reservoir for Leishmania tropica s.l. in Kenya. Ann. Trop. Med. Parasitol. 86: 311-2.

Shehata, MG, Samy, AM, Doha, SA, Fahmy, AR, Kaldas, RM, et al, 2009: First report of Leishmania tropica from a classical focus of $L$. major in North-Sinai, Egypt. Am. J. Trop. Med. Hyg. 81, 2:213-8.

Smart, J, Jordan, K, Whittick, R, 1965: Insect of medical importance, $4^{\text {th }}$ ed., British Museum Natural History, Adlen Press Oxford.

Soliman, MF, 2006: The persistence, dissemination, and visceralization tendency of Leishmania major in Syrian hamsters. Acta Trop. 97:14650 .

Svobodova, M, Votypka, J, Peckova, J, Dvorak, V, Nasereddin, A, et al, 2006: Distinct transmission cycles of Leishmania tropica in 2 adjacent foci, Northern Israel. Emerg. Infect. Dis. 12:1860-8.

Kassem, HA, Siri, J, Kamal, HA, Wilson, ML, 2012: Environmental factors underlying spatial patterns of sand flies (Diptera: Psychodidae) associated with leishmaniasis in southern Sinai, Egypt. Acta Trop. 123, 1:8-15.

Talmi-Frank, D, Jaffe, CL, Nasereddin, A, Warburg, A, King, R, et al, 2010: Leishmania tropica in rock hyraxes (Procavia capensis) in a focus of human cutaneous leishmaniasis. Am. J. Trop. Med. Hyg. 82, 5:814-8.

Tamura, K, Stecher, G, Peterson, D, Filipski, A, Kumar S, 2013: MEGA6: Molecular Evolutionary Genetics Analysis version 6.0. Mol. Biol. Evol. 30:2725-9.

Wahba, MM, Schnur, LF, Morsy, TA, Merdan, A, 1990: The characterization of Leishmania major from Phlebotomus papatasi (Scopoli) caught in northern Sinai, Egypt. Trans. R. Soc. Trop. Med. Hyg. 84, 6:785-6.

Wasserberg, G, Abramsky, Z, Anders, G, ElFari, M, Schoenian, G, et al, 2002: The ecology of cutaneous leishmaniasis in Nizzana, Israel: infection patterns in the reservoir host, and epidemiological implications. Int. J. Parasitol. 32: 133-43.

WHO, 2010: Control of the leishmaniasis. Tech. Rept. Ser. No. 949, Geneva. 